\title{
Effectiveness of a prevention of mother-to-child HIV transmission program in Guangdong province from 2007 to 2010
}

\author{
Bing Li ${ }^{1,2}$, Qingguo Zhao ${ }^{2}$, Xiaozhuang Zhang ${ }^{2}$, Li Wu², Tingting Chen², Zhijiang Liang ${ }^{2}$, Longchang Xu ${ }^{2}$ \\ and Shouyi $Y u^{1 *}$
}

\begin{abstract}
Background: To achieve the goal of United Nations of elimination of new HIV infections, a program of prevention of mother-to-child transmission (PMTCT) was launched in Guangdong province. The objective of this study is to evaluate the effectiveness of the PMTCT program.

Methods: The retrospective cross-section analysis was conducted using the data of case reported cards of HIV positive mothers and their infants from 2007 to 2010 in Guangdong province, and 108 pairs of eligible subjects were obtained. We described the data and compared the rates of MTCT by various PMTCT interventions respectively.

Results: The overall rate of HIV MTCT was 13.89\% (15) among 108 pairs of HIV positive mothers and their infants; $60.19 \%$ (65) of the mothers ever received ARVs, $80.56 \%$ (87) of infants born to HIV positive mothers ever received ARVs, but $16.67 \%$ (18) of the mothers and infants neither received ARVs. Among all the mothers and infants, who both received ARVs, received triple ARVs, mother received ARVs during pregnancy, and both received ARVs and formula feeding showed the lower rates of HIV MTCT, and the rates were $8.06 \%, 2.50 \%, 5.77 \%$, and $6.67 \%$ respectively. In infants born to HIV positive mother, who received mixed feeding had a higher HIV MTCT up to 60.00\%. Delivery mode might not relative to HIV MTCT.

Conclusions: The interventions of PMTCT program in Guangdong could effectively reduce the rate of HIV MTCT, but the effectiveness of the PMTCT program were heavily cut down by the lower availability of the PMTCT interventions.
\end{abstract}

Keywords: HIV/AIDS, Mother to children transmission (MTCT), Anti-retroviral (ARV), Effectiveness of PMTCT

\section{Background}

Mother-to-child transmission (MTCT) of human immunodeficiency virus (HIV) is the most significant route of HIV infection in children. At the United Nations 2011 High Level Meeting on AIDS, leaders committed to achieve the goal of eliminating new HIV infections among children by 2015 [1,2]. According to Estimation on China AIDS Epidemic in 2011 issued by Ministry of Health, there were an estimated 780000 (620 000-940 000)

\footnotetext{
* Correspondence: 522276188@qq.com

'Department of Epidemiology, School of Public Health and Tropical Medicine, Southern Medical University, Guangzhou North Road 1838, Guangzhou 510515, China

Full list of author information is available at the end of the article
}

survival people living with HIV (PLHIV), 28.6\% of PLHIV were female, and $1.1 \%$ of PLHIV were infected by MTCT, there was a higher HIV prevalence among pregnant women (the highest prevalence more than $1 \%$ ) in severely epidemic region [3], these data demonstrate that we face a huge challenge of Prevention of Mother-to-Child Transmission (PMTCT) in China.

However, without intervention, MTCT occurs in up to 4 of every 10 deliveries among HIV-positive women [4]. Without treatment, approximately half of the children who have acquired HIV die before two years of age, and very few survive their school years $[4,5]$. Due to different capability, resource and availability of health service, different region had a different result of PMTCT; the rate

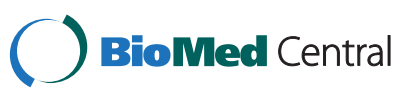

(c) 2013 Li et al.; licensee BioMed Central Ltd. This is an Open Access article distributed under the terms of the Creative Commons Attribution License (http://creativecommons.org/licenses/by/2.0), which permits unrestricted use, distribution, and reproduction in any medium, provided the original work is properly cited. 
of MTCT could be reduced to $5-8 \%$ by integrated PMTCT program in developing country, and it could be reduced to less than $2 \%$ in some developed countries [6-8]. Guangdong is one of the higher HIV prevalence provinces in China [3], and it has initiated to implement free PMTCT program in some districts since 2003. However, the effectiveness of this program in the field are scarcely known. In order to explore this, we evaluated the effectiveness of PMTCT program with evaluating the rates of MTCT by various PMTCT interventions respectively.

\section{Method}

\section{Study design}

The retrospective cross-section analysis was conducted using the data of reported case cards of HIV positive mothers and their infants from 2007 to 2010 in Guangdong province of China. The case cards of HIV positive mothers were reported in five days after their HIV infected status were confirmed, and then the case cards of all infants born to HIV positive mothers were reported in five days after their HIV infected status were tested in the follow up period of 18 months. The mother's HIV infected status was confirmed by the Western blot after her HIV antibody was positive. The infant's HIV infected status was confirmed by the Western blot after the HIV antibody was positive at age 12 months and 18 months. If the condition was available, the infant's HIV infected status was confirmed by HIV DAN polymerase chain reaction (PCR) tests through transported dry blood spots (DBS) at age 6 weeks to 3 months. During the follow up period, there were no death of infants, and only four infants became AIDS patients.

The targeted subjects needed to meet four requirements: 1) Born in hospital of Guangdong province from 2007 to 2010; 2) The mother's HIV infected status was confirmed; 3) The infant born to HIV infected mother was followed up until to 18 months age and the infant's HIV infected status was confirmed; 4) The data of ARV prophylaxis or treatment, delivery mode, and feeding were completely. We obtained 108 pairs of eligible HIV positive mothers and infants from 196 pairs of them in this article. We described the status of subjects of age, marriage status, education level, occupation, and ARV prophylaxis, and we compared the rates of MTCT respectively by various PMTCT interventions, such as ARV prophylaxis, delivery mode, and feeding mode.

\section{Programm description}

The key interventions of the free PMTCT program included general health education on PMTCT, and providerinitiated testing and counseling (PITC) during pregnancy, safe and in hospital delivery and anti-retroviral (ARV) prophylaxis or treatment for HIV positive mothers, and formula feeding and ARV prophylaxis for infants born to HIV-positive mothers. If the HIV infected status confirmed antematally and the service condition of caesarian delivery was safe, the pregnancy women were planed to elective caesarian delivery.

With the consideration of availability of ARV drugs and existed evidences, and reference of regimen recommend by WHO since 2004, the PMTCT program of Guangdong from 2007 to 2010 used the ARV prophylaxis protocol of zidovudine (AZT) started in late pregnancy, combined with lamivudine (3TC) and single-dose nevirapine (sdNVP) during labour, and stopped in one week after delivery. And women with a CD4 count below 200 cells $/ \mathrm{mm} 3$ were given antiretroviral treatment. Due to any unexpected reasons, if the HIV positive mothers couldn't take the ARVs before parturient, the mothers would be offered sdNVP intrapartum as soon as possible.

\section{Statistic analysis}

The descriptive analysis was conducted, and the mean and standard deviation were used to describe the measurement variables, and the frequency distribution was used to describe the categorical variable. The statistical significance of the comparison of proportions was determined using $\chi^{2}$ or Fisher's exact test. The OR values and their 95\% confidence intervals of the factors were estimated. The bivariate correlate was used to analyze the relationship between confirmed period of HIV infected status and whether received ARVs of mothers, and the Spearman correlation coefficient was estimated. All the statistical analyzes were done with SPSS version 13.

\section{Results}

\section{Participant characteristics}

Among $108 \mathrm{HIV}$ positive mothers, the mean age was $27.21 \pm 5.00$ years, the mean numbers of pregnancies were $3.12 \pm 1.25$, the mean delivery times were $1.82 \pm 0.93$; Regarding marital status, $77.78 \%$ were first marriage, $12.04 \%$ were remarriage, $2.78 \%$ were cohabitation, $7.41 \%$ were unmarried; The education degree was lower, $49.07 \%$ were junior middle school, $27.78 \%$ were senior middle school, $15.74 \%$ were primary school, and university or college education and beyond only accounted $4.63 \%$; The majority of occupations (64.81\%) were household duties or unemployed, 9.26\% were workers, 9.26\% were farmers, $4.63 \%$ were other occupations, and $4.63 \%$ were unknown. The detail data reference Table 1.

Among 108 infants born to HIV positive mother, $58.3 \%$ (63) were male, and $41.7 \%$ (45) were female, and the mean birth gestation age was $38.38 \pm 1.81$ weeks, and $90.74 \%$ (98) of them born at or after 37 gestation weeks. 
Table 1 Characteristics of pregnant HIV-infected women

\begin{tabular}{|c|c|c|}
\hline Maternal characteristics & n & $\%$ \\
\hline \multicolumn{3}{|l|}{ Maternal age,median (range) } \\
\hline \multicolumn{3}{|l|}{ Marital status } \\
\hline First marriage & 84 & 77.78 \\
\hline Remarriage & 13 & 12.04 \\
\hline Cohabitation & 3 & 2.78 \\
\hline Unmarried & 8 & 7.41 \\
\hline \multicolumn{3}{|l|}{ Education } \\
\hline Primary school & 17 & 15.74 \\
\hline Junior middle school & 53 & 49.07 \\
\hline Senior middle school & 30 & 27.78 \\
\hline College degree or above & 5 & 4.63 \\
\hline Illiteracy & 2 & 1.85 \\
\hline Unknown & 1 & 0.93 \\
\hline \multicolumn{3}{|l|}{ Occupation } \\
\hline Household duties or unemployed & 70 & 64.81 \\
\hline Workers & 10 & 9.26 \\
\hline Farmers & 10 & 9.26 \\
\hline Others & 13 & 12.04 \\
\hline Unknown & 5 & 4.63 \\
\hline \multicolumn{3}{|l|}{ Parity } \\
\hline Primiparity & 49 & 45.37 \\
\hline Multiparity & 59 & 54.63 \\
\hline
\end{tabular}

\section{ARV prophylaxis}

In all of 108 HIV positive mothers, only $60.19 \%$ (65) of them ever received ARVs prophylaxis from 28 weeks to one week of postpartum, but only $37.04 \%$ (40) of mothers received triple ARVs regimen.

In the $52(48.15 \%, 52 / 108)$ mothers who received ARVs prophylaxis antenatally, 59.62\% (31) received AZT, 26.92\% (14) received sdNVP+3TC+AZT, 3.85\% (2) received 3TC+AZT.

In the $60(55.56 \%, 60 / 108)$ mothers who received ARVs prophylaxis intrapartum, 66.67\% (40) received sdNVP+ 3TC+AZT, $15.00 \%$ (9) received sdNVP+AZT, $10.00 \%$ (6) received AZT, $8.33 \%(5)$ received $3 \mathrm{TC}+\mathrm{AZT}$.

In the $56(51.85 \%, 56 / 108)$ mothers who received ARVs prophylaxis postnatal, $48.21 \%$ (27) were $3 \mathrm{TC}+\mathrm{AZT}$, $30.36 \%$ (17) were sdNVP+3TC+AZT, $17.86 \%$ (10) were AZT, $1.79 \%$ (1) were sdNVP+AZT, $1.79 \%$ (1) were 3TC.

There were $80.56 \%(87 / 108)$ of infants born to HIV positive mothers received ARVs prophylaxis, and in these 87 infants, $77.01 \%$ (67) of them received sdNVP+AZT, $10.34 \%$ (9) received AZT, 8.05\% (7/87) received sdNVP, $3.45 \%$ (3) received $3 \mathrm{TC}+\mathrm{AZT}, 1.15 \%$ (1) received $3 \mathrm{TC}+$ sdNVP+AZT.

Among 108 pairs of HIV positive mothers and their infants, $16.67 \%$ (18) of them neither received ARVs,
$2.78 \%$ (3) of them only mothers received ARVs, $23.15 \%$ (25) of them only infants received ARVs, $57.41 \%$ (62) of them both received ARVs.

\section{Confirmed period of HIV infected status of mothers}

As to the confirmed period of HIV infected status of 108 mothers, $5.56 \%$ (6) were before the present pregnancy, $53.70 \%$ (58) were antenatal of this pregnancy, $18.52 \%$ (20) were in the intrapartum of this pregnancy, $22.22 \%$ (24) were in the postpartum of this pregnancy. The proportion of non-received ARVs of mothers who confirmed HIV infected status before the present pregnancy, antenatal, intrapartum, and postpartum was respectively $33.33 \%(2 / 6)$, 8.62\% (5/58), 75.00\% (15/20) and 87.50\% (21/24). There was a correlation between confirmed period of HIV status and whether received ARVs, and the Spearman correlation coefficient was $0.66(\mathrm{~T}=8.97, \mathrm{P}<0.001)$, which illustrated more later confirmed HIV infected status was more less possibly received ARVs of mothers.

\section{The mode of delivery and feeding}

Among 108 pairs of mothers and infants, 22.22\% (24) of infants delivered by emergency C-section, $45.37 \%$ (49) of infants delivered by vagina, and $32.41 \%$ (35) of infants delivered by elective C-section; Formula feeding was 91.67\% (99), breast feeding was 3.70\% (4), and mixed feeding was $4.63 \%$ (5). There were $55.56 \%$ (60) of them both mothers and infants received ARVs and formula feeding, and $24.07 \%$ (26) of them received all the four interventions including both mothers and infants received ARVs, elective C-section, and formula feeding.

\section{The effect of received ARVs, delivery mode, and feeding type on MTCT of HIV}

The overall rate of HIV MTCT was 13.89\% (15) among 108 infants born to HIV positive mothers. The data in Table 2 showed that in all the mothers and infants, who both received ARVs, received triple ARVs, mother received ARVs antenatally, and both received ARVs and formula feeding showed the lower rates of HIV MTCT, and the rates were $8.06 \%, 2.50 \%, 5.77 \%$, and $6.67 \%$ respectively. In infants born to HIV positive mother, who received mixed feeding had a higher HIV MTCT up to $60.00 \%$. Delivery mode might not relative to HIV MTCT. MTCT of HIV was eliminated among the 26 subjects who received all the four interventions including both mothers and infants received ARVs, elective Csection, and formula feeding.

The rate of HIV MTCT of infants whose mothers ever received ARVs and non-received ARVs was 9.23\% (6/65) and $20.93 \%(9 / 43)$ respectively ( $p>0.05)$, and that of infants who received and non-received ARVs was 10.34\% $(9 / 87)$ and $28.57 \%(6 / 21)$ respectively ( $p>0.05)$. 
Table 2 The relationships between ARVs, delivery mode or feeding type and MTCT of HIV $(n=108)$

\begin{tabular}{|c|c|c|c|c|c|c|}
\hline \multirow[t]{2}{*}{ Interventions of PMTCT } & \multicolumn{2}{|c|}{ Infants' HIV test results } & \multirow[t]{2}{*}{ Total } & \multirow[t]{2}{*}{$x^{2}$ value } & \multirow[t]{2}{*}{$P$-value } & \multirow[t]{2}{*}{$\mathrm{OR}(95 \% \mathrm{Cl})$} \\
\hline & $\begin{array}{l}\text { Positive } \\
\mathrm{N}(\%)\end{array}$ & $\begin{array}{l}\text { Negative } \\
\mathrm{N}(\%)\end{array}$ & & & & \\
\hline Who received ARVs & & & & Fisher's Exact & 0.07 & \\
\hline Neither & $5(27.78)$ & 13(72.22) & 18 & & & $4.38(1.11,17.40)$ \\
\hline Mother only or infant only & $5(17.86)$ & 23(82.14) & 28 & & & $2.48(0.65,9.38)$ \\
\hline Both & $5(8.06)$ & $57(91.94)$ & 62 & & & 1.0 \\
\hline Mother's ARVs regimen & & & & 6.90 & 0.032 & \\
\hline None any ARVs & $9(20.93)$ & $34(79.07)$ & 43 & & & $10.32(1.24,85.71)$ \\
\hline Non-triple ARVs & $5(20.00)$ & $20(80.00)$ & 25 & & & $9.75(1.07,89.20)$ \\
\hline Triple ARVs & $1(2.50)$ & $39(97.50)$ & 40 & & & 1.0 \\
\hline Mother received ARVs antenatally & & & & 5.53 & 0.02 & $4.45(1.18,16.83)$ \\
\hline Yse & $3(6.12)$ & 49(94.23) & 52 & & & \\
\hline No & 12(27.27) & $44(78.57)$ & 56 & & & \\
\hline Mother and infant both received ARVs and formula feeding & & & & 6.51 & 0.039 & \\
\hline None of three & 2(33.33) & $4(66.67)$ & 6 & & & 7.0(0.97,50.57) \\
\hline One or two of three & $9(21.43)$ & $33(78.57)$ & 42 & & & $3.82(1.09,13.38)$ \\
\hline All of three & $4(6.67)$ & $56(93.33)$ & 60 & & & 1.0 \\
\hline $\begin{array}{l}\text { Mother and infant both received ARVs and formula feeding and } \\
\text { elective C-section }\end{array}$ & & & & $\begin{array}{l}\text { Fisher's Exact } \\
\text { Test }\end{array}$ & 0.01 & \\
\hline None of four & 2(33.33) & $4(66.67)$ & 6 & & & $1.50(0.85,2.64)$ \\
\hline One to three of four & 13(17.11) & 63(82.89) & 76 & & & $1.21(1.09,1.34)$ \\
\hline All of four & $0(0.00)$ & $26(100.00)$ & 26 & & & 1.0 \\
\hline Feeding type & & & & Fisher's Exact & 0.04 & \\
\hline Mixed feeding & $3(60.00)$ & $2(40.00)$ & 5 & & & $10.88(1.65,71.86)$ \\
\hline Breast feeding & $0(0.00)$ & $4(100.00)$ & 4 & & & $0.88(0.82,0.95)$ \\
\hline Formula feeding & $12(12.12)$ & $87(87.88)$ & 99 & & & 1.0 \\
\hline Delivery mode & & & & Fisher's Exact & 0.49 & \\
\hline Emergency C-section & $3(12.50)$ & $21(87.50)$ & 24 & & & $0.63(0.16,2.60)$ \\
\hline Vagina delivery & $3(8.57)$ & $32(91.43)$ & 35 & & & $0.42(0.10,1.67)$ \\
\hline Elective C-section & $9(18.37)$ & $40(81.63)$ & 49 & & & 1.0 \\
\hline Total & 15(13.89) & 93(86.11) & 108 & & & \\
\hline
\end{tabular}

\section{Discussion}

The study showed that the overall rate of HIV MTCT was $13.89 \%$ in Guangdong province from 2007 to 2010 after PMTCT interventions were implemented, and it was a MTCT rate of HIV similar to resource-limited region [8-10], and did not achieve the expectant goal of reducing the rate of HIV MTCT down to less than $5 \%$. One of the main reasons of un-achieved the goal was the lower availability of the PMTCT interventions. The data of this article showed a lower and out-timely availability of ARVs of HIV positive mothers and their infants. Only $60.19 \%$ of HIV positive mothers received ARVs, and only $48.15 \%$ of them received ARVs during pregnancy, and merely $60.19 \%$ of them received triple ARVs regimen, and $80.56 \%$ of infants born to them received ARVs, and the formula feeding rate was $91.67 \%$, and only $55.56 \%$ of mothers and their infants both received ARVs and formula feeding.

Consideration the conditions and surroundings of the PMTCT program, maybe many causes resulted in the lower availability of the PMTCT interventions. Such as, not enough HIV test ability of primary health service institutions, not enough training on PMTCT for primary medical personnel, HIV positive mothers initially participated antenatal care lately and even until to nearly delivery, following up difficulty for flowing population and worrying about discrimination, pregnant women do not know about the PMTCT interventions, and the ARVs for infants need to be imported and can't be provided timely. A lot of literatures reported that many influencing factors can result in 
missing opportunities for prevention of mother-to-child transmission of HIV among HIV positive mothers and their infants, such as people's knowledge about PMTCT, the antenatal service system and HIV test ability of facilities, following up mode [11-17]. The data of this study showed that $18.52 \%$ of HIV positive mothers confirmed HIV infected status during delivery, and $22.22 \%$ of them confirmed HIV infected status postnatal, and that resulted in lower availability of ARVs.

Although there was no significant difference of overall HIV-MTCT rate between mothers or infants received $A R V s$ and those non-received ARVs, the data showed that mothers and their infants neither received ARVs compared to those both received ARVs, mothers received non-triple ARVs compared to those received triple ARVs, and mothers non-received ARVs during pregnancy compared to those received ARVs during pregnancy, can increase the HIV-MTCT rate, and the OR value was 4.38 (95\% CI: 1.11-17.40), 9.75 (95\% CI: 1.07-89.20) and 4.32 (95\% CI: 1.09-17.15) respectively. If HIV positive mothers receive ARVs following the referred regimen, the HIV-MTCT rate could be decreased to $2.5 \%$; and if HIV positive mothers received ARVs during pregnancy, the HIV-MTCT rate could be decreased to $5.77 \%$, and the effects of PMTCT were similar to that in developed countries $[8,10,18,19]$. The data illustrated that HIV positive mothers and their infants received ARVs timely and routinely can help to decrease the HIV MTCT rate significantly, and the helping was limited if they received ARVs out-time or un-routinely.

The data of this study showed that mixed feeding increased the opportunity of HIV-MTCT compared to formula feeding $(\mathrm{OR}=10.88,95 \% \mathrm{CI}: 1.65-71.86)$, and decreased the opportunity of HIV-MTCT compared to breast feeding (OR=0.88, 95\% CI: 0.82-0.95)). The study of Gerardo et al. revealed that universal ARV therapy to HIV-infected pregnant women is able to reduce motherto-child transmission to less than 5\%, although breastfeeding can increase the rate of HIV transmission, formula feeding produces malnutrition during the first month of life, increases mortality compared to breastfeeding [20]. The results of whether breastfeeding increased the rate of HIV MTCT was different, but only 4 infants received breastfeeding in our data, it needed more samples to be further analyzed.

Siobhan et al. reported that there was no association between mode of delivery and HIV MTCT, which was consistent with our findings [21]. But we found that when HIV positive mothers and their infants received ARVs and formula feeding, and plus elective $\mathrm{C}$-section could further reduce HIV MTCT. Mothers and their infants both received ARVs and formula feeding could reduce HIV MTCT down to 6.67\% (4/60), but HIV MTCT was eliminated in 26 mothers and their infants who received all the interventions including received ARVs, elective $\mathrm{C}$-section, and formula feeding.

The review of Tudor et al. described that integrated PMTCT services could reduce MTCT effectively [22]. The study of Ngozi et al. showed that holistic but cost effective preventive interventions help in reducing the rate of mother-to-child transmission of HIV even in economicallydeveloping settings [23]. Turan et al. found that PMTCT of HIV and antenatal care integration in pregnancy might improve the implementation and effectiveness of PMTCT in rural area [24]. All of above mentioned illustrated that it was important to strengthen the implementation of PMTCT program to increase the availability of interventions in Guangdong province for achieving the goal of elimination of new infections among children by 2015 , which was similar to the problems of developing country [25].

A number of clinical trials have showed that MTCT can now be reduced to less than 2 percent from a possible 2530 percent without any intervention [17]. With the cumulation of evidence of PMTCT, the recommendation protocol of PMTCT is kept on update. The finding of this study denominated that the protocol of PMTCT program in Guangdong from 2007 to 2010 could effectively reduce the rate of MTCT, but that effectiveness were heavily cut down by the lower availability of integrated PMTCT interventions. Although recently the evidence showed that the breastfeeding could be use for PMTCT in many developing countries, but the formula-feeding was more benefit to PMTCT in the setting of Guangdong province.

In addition, the data of this article showed that there was no significant decrease of HIV MTCT rate in the funded PMTCT regions compared to non-funded PMTCT regions (11.29\% vs. $17.39 \%)$ after the PMTCT program was launched in Guangdong. And the loss to follow-up among infants was the main obstacle of effective evaluation of PMTCT program, there were 196 pairs of HIV positive mothers and their infants were reported form 2007 to 2010 in Guangdong, only 55.1\% $(108 / 196)$ of them were eligible. All of the excluded objects were due to loss to follow-up the HIV infectious status of infants, which mostly because of population flowing and the parent was afraid of impact to the life of their child after the HIV infectious status was exposed. According to the information of mothers' reported cards, in 88 subjects who lost to follow up, the mean age of mother was $28.78 \pm 5.28$ years, which was higher than the mean age of targeted subject $(t=2.13, p=0.03)$; But the distributions of marital status, education level, occupation, and the rate of ARV prophylaxis were no significant difference respectively between lost follow up mothers and targeted mothers ( $>>0.05)$. And 67.05\% (59/88) of mothers and 79.55\% (70/88) of their infants ever received ARVs among 88 subjects who lost to 
follow up. According to these data, it was suppose that the estimation of MTCT rates in this study was acceptable.

\section{Conclusions}

At present, the data showed that the overall rate of HIV MTCT was $13.89 \%$ in Guangdong province from 2007 to 2010 after PMTCT interventions were implemented, which did not achieve the expectant goal of reducing the rate of HIV MTCT down to less than 5\%. But in the HIV positive mothers and their infants who could receive the interventions recommended by PMTCT program, the rate of HIV MTCT might be reduced down to below 5.0\%. Therefore, the interventions of PMTCT program in Guangdong could effectively reduce the rate of HIV MTCT, but the effectiveness of the PMTCT program were heavily cut down by the lower availability of the PMTCT interventions.

\section{Ethical consideration}

Ethical approval was obtained from Guangdong Women and Children Hospital Ethics Committee.

\section{Competing interests}

The authors declare that they have no competing interests.

\section{Authors' contributions}

LB performed the statistical analysis and drafted the manscript; $L J, X C$ and $C T$ collected the data. ZG, ZZ and $Y Y$ conceived the study; $Y Y$ and $W L$ revised the mansucript. All authors read and approved the final manuscript.

\section{Acknowledgments \\ The authors would like to thank Hu Ting-ting and Huang Yan-hui for the help in writing formation, and thank all the workers of data collection in all levels of Maternal and Children Hospitals in Guangdong, and thank the teachers of Department of Epidemiology of School of Public Health and Tropical Medicine of Southern Medical University for instruction and copyediting the manuscript.}

\section{Author details}

'Department of Epidemiology, School of Public Health and Tropical Medicine, Southern Medical University, Guangzhou North Road 1838, Guangzhou 510515, China. ${ }^{2}$ Guangdong Women and Children Hospital, Xingnan Road 521, Guangzhou 511400, China.

Received: 19 November 2012 Accepted: 26 April 2013

Published: 18 June 2013

\section{References}

1. WHO and UNICEF with the Inter-Agency Task Team: Guidance on global scale-up of the prevention of mother-to-child transmission of HIV: towards universal access for women, infants and young children and eliminating HIV and AIDS among children. Geneva: WHO; 2007.

2. UNAIDS: Joint action for results: UNAIDS outcome framework, 2009-2011. Geneva: UNAIDS; 2010

3. Ministry of Health, People's Republic of China, Joint United Nations Program on HIV/AIDS, World Health Organization: 2011 Estimates for the HIV/AIDS Epidemic in China. Beijing: National Center for AIDS/STD Control and Prevention of China CDC; 2011. http://www.moh.gov.cn/mohjbyfkzj/s3586/ 201201/53957.shtml.

4. UNAIDS: Report on the Global AIDS Epidemic 2010. Geneva: UNAIDS; 2010

5. Marcos $Y$, Ryan Phelps B, Bachman G: Community strategies that improve care and retention along the prevention of mother-to-child transmission of HIV cascade: a review. J Int AIDS Soc 2012, 15(Suppl 2):17394.
6. Brocklehurst $P$ : Interventions for reducing the risk of mother-to-child transmission of HIV Infection. Cochrane Database Syst Rev 2002, 1, CD000102

7. Dabis F, Newell ML, Fransen L, Saba J, Lepage P, Leroy V, Cartoux M, Meda N, Whynes DK, Peckham C, Nduati R, Msellati P, Vincenzi ID, van de Perre P. Prevention of mother to child transmission of HIV in developing countries: recommendations for practice. Health Policy Plan 2000, 15:34-42.

8. WHO, UNAIDS: Preventing mother-to-child transmission of HIV to reach the UNGASS and Millennium Development Goals. Geneva 27, Switzerland: PMTCT Strategic Vision 2010-2015. 20 Avenue Appia, 1211; 2010.

9. De Cock KM, Fowler MG, Mercier E, de Vincenzi I, Saba J, Hoff E, Alnwick DJ, Rogers M, Shaffer N: Prevention of mother-to-child HIV transmission in resource-poor countries: translating research into policy and practice. JAMA 2000, 283:1175-1182.

10. Coetzee D, Hilderbrand K, Boulle A, Draper B, Abdullah F, Goemaere E: Effectiveness of the first district-wide programme for the prevention of motherto-child transmission of HIV in South Africa. Bull World Health Organ 2005, 83:489-494.

11. Peltzer K, Sikwane E, Majaja M: Factors associated with short-course antiretroviral prophylaxis (dual therapy) adherence for PMTCT in Nkangala district, South Africa. Acta Paediatr 2011, 100(9):1253-1257.

12. Torpey K, Mandala J, Kasonde P, Bryan-Mofya G, Bweupe M, Mukundu J, Zimba C, Mwale C, Lumano H, Welsh M: Analysis of HIV early infant diagnosis data to estimate rates of perinatal HIV transmission in Zambia. PLoS One 2012, 7(8):e42859.

13. Read JS, Cohen RA, Hance LF, Machado ES, Mussi-Pinhata MM, Ceriotto M, Santos B, Succi R, Pilotto JH, Alarcon JO, Kreitchmann R: Missed opportunities for prevention of mother-to-child transmission of HIV-1 in the NISDI Perinatal and LILAC cohorts. Int J Gynaecol Obstet 2012, 119(1):70-75.

14. Barker PM, Mate K: Eliminating Mother-To-Child HIV transmission will require major improvements in maternal and child health services. Health Aff (Millwood) 2012, 31(7):1489-1497.

15. Wettstein C, Mugglin C, Egger M, Blaser N, Vizcaya LS, Estill J, Bender N, Davies MA, Wandeler G, Keiser O: Missed opportunities to prevent Mother-to-Child transmission in sub-Saharan Africa: systematic review and meta-analysis. AIDS 2012, 26(18):2361-2373.

16. Larsson EC, Thorson AE, Pariyo G, Waiswa P, Kadobera D, Marrone G, Ekström AM: Missed Opportunities: Barriers to HIV testing during pregnancy from a population based cohort study in rural Uganda. PLoS One 2012, 7(8):e37590.

17. Chetty T, Knight S, Giddy J, Crankshaw TL, Butler LM, Newell ML: A retrospective study of Human Immunodeficiency Virus transmission, mortality and loss to follow-up among infants in the first 18 months of life in a prevention of mother-to-child transmission program in an urban hospital in KwaZulu-Natal, South Africa. BMC Pediatrics 2012, 12:146.

18. Torpey K, Kasonde P, Kabaso M, Weaver MA, Bryan G, Mukonka V, Bweupe M, Zimba C, Mwale F, Colebunders R: Reducing pediatric HIV infection: estimating mother-to-child transmission rates in a program setting in Zambia. J Acquir Immune Defic Syndr 2010, 54(4):415-422.

19. Torpey K, Kasonde P, Kabaso M: Reducing pediatric HIV infection: estimating mother-to-child transmission rates in a program setting in Zambia. J Acquir Immune Defic Syndr 2010, 54(4):415-422.

20. Alvarez-Uria G, Midde M, Pakam R, Bachu L, Naik PK: Effect of formula feeding and breastfeeding on child growth, infant mortality, and HIV transmission in children born to HIV-infected pregnant women Who received triple antiretroviral therapy in a resource-limited setting: data from an HIV cohort study in India. ISRN Pediatrics 2012, 201(2):763591.

21. Mark S, Murphy KE, Read S, Bitnun A, Yudin MH: HIV mother-to-child transmission, mode of delivery, and duration of rupture of membranes: experience in the current Era. Infect Dis Obstet Gynecol 2012, 201(2):267969.

22. Tudor Car L, van-Velthoven MH, Brusamento S, Elmoniry H, Car J, Majeed A Tugwell P, Welch V, Marusic A, Atun R: Integrating prevention of motherto-child HIV transmission programs to improve uptake: a systematic review. PLoS One 2012, 7(4):e35268.

23. Ibeziako NS, Ubesie AC, Emodi IJ, Ayuk AC, Iloh KK, Ikefuna AN: Mother-tochild transmission of HIV: the pre-rapid advice experience of the university of Nigeria teaching hospital Ituku/Ozalla, Enugu, South-east Nigeria. BMC Research Notes 2012, 5:305. 
24. Turan JM, Steinfeld RL, Onono M, Bukusi EA, Woods M, Shade SB, Washington S, Marima R, Penner J, Ackers ML, Mbori-Ngacha D, Cohen CR: The study of HIV and antenatal care integration in pregnancy in Kenya: design, methods, and baseline results of a cluster-randomized controlled trial. PLoS One 2012, 7(9):e44181.

25. Darak S, Panditrao M, Parchure R, Kulkarni V, Kulkarni S, Janssen F:

Systematic review of public health research on prevention of mother-tochild transmission of HIV in India with focus on provision and utilization of cascade of PMTCT services. BMC Publ Health 2012, 12:320.

doi:10.1186/1471-2458-13-591

Cite this article as: Li et al:: Effectiveness of a prevention of mother-tochild HIV transmission program in Guangdong province from 2007 to 2010. BMC Public Health 2013 13:591.

\section{Submit your next manuscript to BioMed Central and take full advantage of:}

- Convenient online submission

- Thorough peer review

- No space constraints or color figure charges

- Immediate publication on acceptance

- Inclusion in PubMed, CAS, Scopus and Google Scholar

- Research which is freely available for redistribution 